\title{
Editorial
}

\section{Anomalous Microwave Emission: Theory, Modeling, and Observations}

\author{
Clive Dickinson, ${ }^{1}$ Roberta Paladini, ${ }^{2}$ and Laurent Verstraete ${ }^{3,4}$ \\ ${ }^{1}$ Jodrell Bank Centre for Astrophysics, School of Physics and Astronomy, The University of Manchester, Oxford Road, \\ Manchester M13 9PL, UK \\ ${ }^{2}$ NASA Herschel Science Center, California Institute of Technology, Pasadena, CA, USA \\ ${ }^{3}$ Univ. Paris-Sud, Institut d'Astrophysique Spatiale, UMR-8617, F-91405 Orsay, France \\ ${ }^{4}$ CNRS, F-91405 Orsay, France \\ Correspondence should be addressed to Roberta Paladini; paladini@ipac.caltech.edu
}

Received 19 September 2013; Accepted 19 September 2013

Copyright ( 2013 Clive Dickinson et al. This is an open access article distributed under the Creative Commons Attribution License, which permits unrestricted use, distribution, and reproduction in any medium, provided the original work is properly cited.

\begin{abstract}
Anomalous Microwave Emission (AME) was first identified in the late 1990s, through sensitive high frequency radio CMB observations. The usual emission mechanisms (e.g., blackbody, synchrotron, and free-free) did not appear to be able to account for the excess emission in the frequency range 10$60 \mathrm{GHz}$. Since then, a large body of observational evidence has emerged showing that AME appears to be emitted both in the diffuse interstellar medium at large, and from specific clouds within our galaxy. Detections from star-forming regions in an external galaxy have also been made. Nevertheless, detailed measurements have been difficult due to the frequency range (difficult to observe from the ground) and confusion with other emission mechanisms that emit in this frequent range. The most promising candidate for the $\mathrm{AME}$ is electric dipole radiation from small spinning dust grains (spinning dust emission). This was first predicted in the late $50 \mathrm{~s}$, with major developments in the theory over the last 15 years. The theory predicts a peaked spectrum which emits at frequencies from about $10 \mathrm{GHz}$ to over $100 \mathrm{GHz}$, but with a wide range of peak frequencies and emissivities, which depend on the local environment and dust grain size distribution. There is still significant debate about the true nature of the AME, and both observations and theory are still relatively unexplored. An exciting possibility is to use detailed radio observations of spinning dust to study the interstellar medium, in a complementary way to the optical, UV, and infrared domains. This special issue is dedicated to the study of AME.
\end{abstract}

E. M. Leitch and A. C. R. Readhead review the discovery of the AME giving further insights into their early work. A detailed review of observations in star-forming regions is given by A. M. M. Scaife, while a detailed theoretical overview of the spinning dust process is explained by Y. Ali-Haïmoud. New theoretical work on the physical effects and implications of grain wobbling is described by T. Hoang and A. Lazarian. E. S. Battistelli et al. discuss the potential and issues involved in using large radio telescopes for AME observations. New observational results and analyses are presented from the Arc Minute Imager (AMI) telescope (Y. C. Perrott et al.), Tenerife experiments (J. F. Macías-Pérez et al.), and WMAP satellite (A. Bonaldi and S. Ricciardi). C. Dickinson summarises observations of AME in HII regions, while C. T. Tibbs et al. discuss the limitation of AME emissivities derived from observations. Finally, J. A. Rubiño-Martín et al. present a comprehensive summary and new results on the polarization of AME.

We hope that researchers from a broad range of astronomical backgrounds (CMB foregrounds, radio astronomy, ISM, and theory) find this special issue useful in bringing the state-of-the-art research in AME into one compendium. We also hope that it may stimulate further research into this fascinating area of astronomy and astrophysics.

Clive Dickinson

Roberta Paladini

Laurent Verstraete 

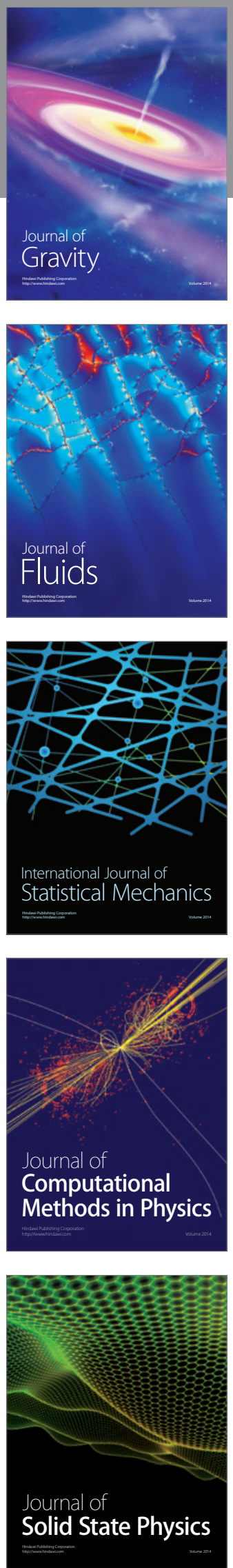

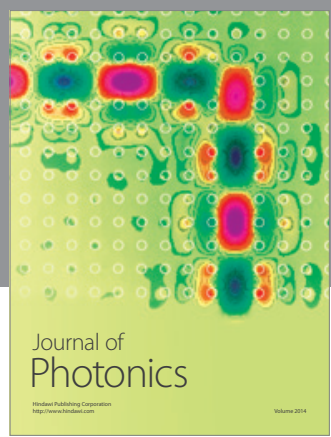

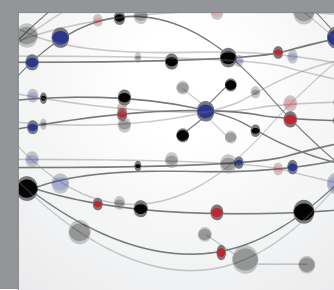

The Scientific World Journal

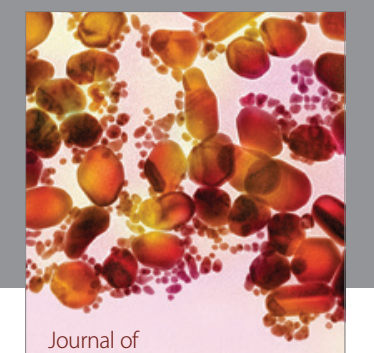

Soft Matter
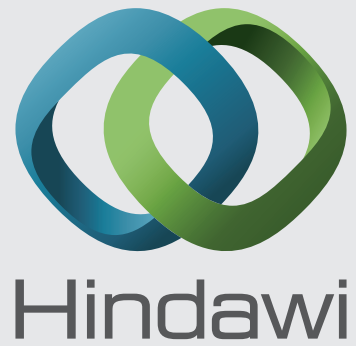

Submit your manuscripts at

http://www.hindawi.com
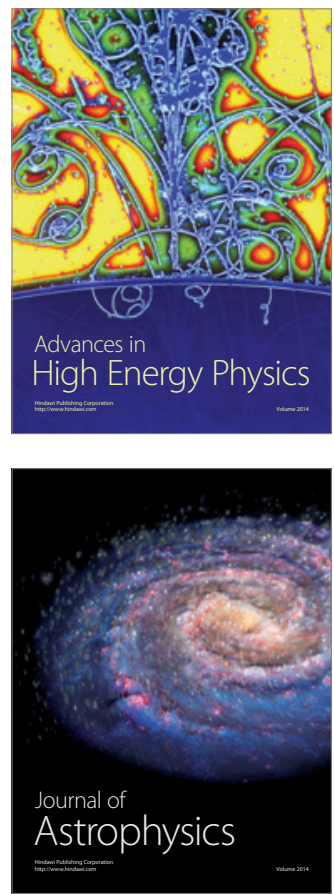
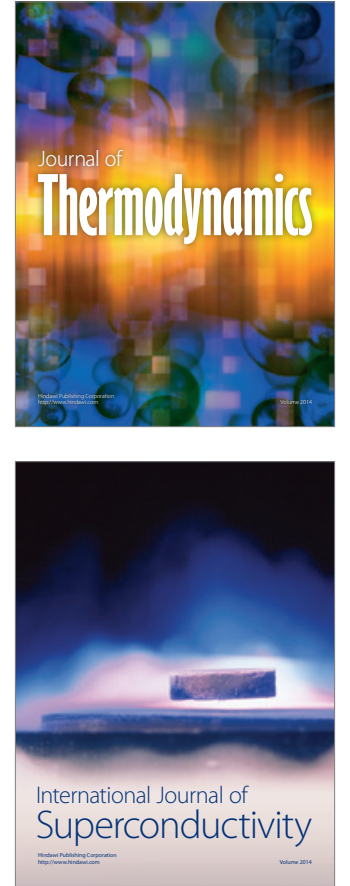
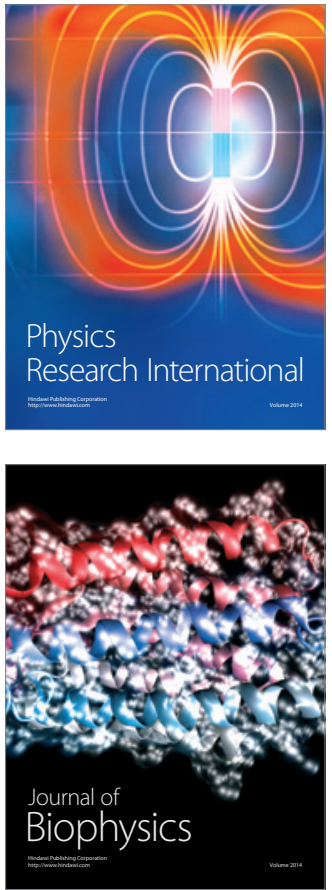
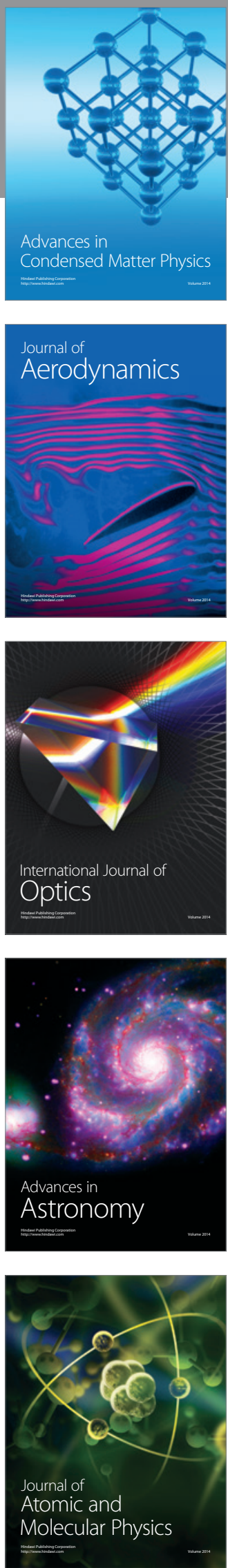